\title{
Costo de las infecciones intrahospitalarias en hospitales chilenos de alta y mediana complejidad
}

\author{
POLA BRENNER F. ${ }^{1}$, PATRICIO NERCELLES M. ${ }^{2,3}$, MÓNICA POHLENZ A. ${ }^{1}$, FERNANDO OTAÍZA O. ${ }^{1}$ \\ y ALUMNOS DEL MAGISTER EN INFECCIONES INTRAHOSPITALARIAS ${ }^{3}$
}

\section{Cost of nosocomial infections in Chilean hospitals}

This paper exhibits the results of 34 cost studies for nosocomial urinary tract infections, wound infections, endometritis, pneumonia, bloodstream infections and caesarean wound infections, conducted in 24 high and medium complexity hospitals in Chile using PAHO (Panamerican Health Organization) proposed methodology: patients with nosocomial infections (NI) were compared with patients without NI of equal sex, age, pathology and clinical unit in hospitals with high and medium complexity compared themselves. The length of stay (LOS) and the use of antimicrobial in DDD were compared in both groups. Conclusion: All studies showed that NI increased length of stay and use of antimicrobial between 2 to 4 times. Wound infections and bloodstream infections increased the LOS more than other NI. Wound infections and pneumonia increased antimicrobial use more than other NI. There were no relevant differences depending on the type of hospitals. The bigger variability was observed in wound infections due probably to the difference in surgical procedures included in the study.

Key words: Nosocomial infection, Hospital infections, Hospital infection cost.

\section{Introducción}

Las infecciones intrahospitalarias (IIH) constituyen una complicación de la atención nosocomial que se ha asociado en numerosas investigaciones con aumento de la morbilidad, mortalidad y costo de los pacientes hospitalizados. Estudios publicados en E.U.A., muestran que en ese país se producen alrededor de 2.000 .000 de IIH anuales y que en promedio presentan alrededor de 5 días de sobre estadía (herida operatoria 7,5 días, bacteriemias 7 a 21 días, neumonía 6,8 a 30 días e infección urinaria 1 a 4 días. Lo anterior significaría 8.676.000 días cama utilizadas en IIH y US\$ 4.532.000.000 ${ }^{1-3}$. En Chile se notifican alrededor de $70.000 \mathrm{IIH}$ anuales y se estima que cada IIH prolonga en promedio 10 días la estadía hospitalaria lo cual significaría 700.000 días cama utilizados en IIH y un costo para el país de US\$ 70.000.0004.

El conocimiento del costo de las IIH en las diferentes realidades, es fundamental para estimar el costo beneficio de los programas de intervención y su impacto en términos económicos. No obstante, la evaluación del costo de las IIH es compleja debido a múltiples factores que pueden incidir en los resultados como: tipo de IIH, agente etiológico y resistencia a los antimicrobianos, complejidad de la atención, tipo de pacientes involucrados y tipo de investigación realizada.

Estimar los verdaderos costos en IIH es complejo y muy dependiente de la metodología utilizada y de los sistemas de atención. En general, la información publicada es parcial, ya que sólo considera los costos directos de la atención como estadía hospitalaria, utilización de antimicrobianos y procedimientos diagnósticos y terapéuticos para enfrentar la IIH. Otros costos tales como secuelas, subsidios, licencias, alteración de la vida familiar y muerte, son difíciles de evaluar en términos económicos.

El diseño más aceptado en la actualidad para

\footnotetext{
Unidad de Infección Intrahospitalaria y Acreditación, Departamento de Calidad de Prestadores, Ministerio de Salud de Chile.

2 Unidad de Epidemiología Hospitalaria, Hospital Carlos Van Buren.

Facultad de Medicina. Universidad de Valparaíso.
} 
estimar costos directos, es el de tipo comparativo, en el cual se comparan las distintas variables en estudio, en pacientes con y sin IIH (de preferencia pareados), de tal manera que se pueda calcular el exceso atribuible a la infección. Para fines comparativos entre instituciones, países e incluso en el tiempo, es más práctico utilizar indicadores no monetarios, como el exceso de días de hospitalización, de reintervenciones quirúrgicas o unidades de antimicrobianos. Por otro lado, una limitación de la mayoría de los estudios de costos, es que sólo contemplan los costos directos de las IIH (asociados a la atención), por la mayor facilidad en obtener esos datos.

En Chile, existen experiencias locales de estudios de costos. En un brote causado por Acinetobacter baumannii en un Servicio de Urología, se produjeron 4 infecciones urinarias y 3 infecciones de herida operatoria. Los resultados indicaron una sobre estadía de 118 días, un exceso de 22 curaciones, 26 cultivos de herida y utilización de $30 \mathrm{~g}$ de amikacina y de $7 \mathrm{~g}$ de sulbactam/ampicilina. En el mismo hospital, se han realizado diferentes estudios de costo de IIH con metodología comparativa. Uno de los estudios mostró que llevando los resultados obtenidos a las IIH notificadas en un año, la sobre estadía hospitalaria correspondió a un total de 13.094 días, lo que equivale a 7\% del total de camas disponibles el mismo año. Las infecciones urinarias produjeron en promedio 12,9 días de exceso de hospitalización; neumonía 18,4 días; herida operatoria 30,5 días; bacteriemia primaria 43,8 días; infecciones asociadas a válvulas derivativas 78,7 días; herida operatoria cesárea 15,6 días y neumonía no asociada a procedimientos invasores 18,1 días $^{5-8}$.

En el año 1999, la Organización Panamericana de la Salud (OPS) como complemento a un programa de monitoreo de la resistencia antimicrobiana, elaboró en Santiago el "Protocolo para determinar el costo de la infección hospitalaria”, basado en métodos comparativos y pareados, para las infecciones más frecuentes en los hospitales, con el fin de disponer de un instrumento homogéneo para realizar estas investigaciones 9.

El objetivo del presente estudio es mostrar los resultados de estudios de costo realizados en diferentes hospitales de alta y mediana complejidad en Chile, utilizando la metodología del protocolo OPS.

\section{Material y Método}

Se incluyeron estudios de costo realizados en el país durante el año 2002 por alumnos del
Magister en Ciencias Médicas con mención en Infecciones Intrahospitalarias y Epidemiología Hospitalaria de la Universidad de Valparaíso, en 24 instituciones de salud desde Antofagasta a Punta Arenas (16 hospitales dependientes del MINSAL, 3 dependientes del Ministerio de Defensa, 3 clínicas privadas y 2 de Mutuales). Los criterios de inclusión fueron el consentimiento del autor y el cumplimiento de los requisitos establecidos en el protocolo. En el protocolo se requiere que el hospital cuente con sistema de vigilancia epidemiológica de IIH con evaluación de la sensibilidad, apoyo microbiológico e información de costos locales. Para la detección de las IIH se utilizaron las definiciones del MINSAL ${ }^{10}$. Se seleccionaron como variables de resultados dos indicadores económicos, el exceso de días de estada y el exceso de uso de antimicrobianos expresados en DDD entre pacientes con IIH y pacientes sin IIH, con un control por cada caso.

Las IIH estudiadas correspondieron a las infecciones más frecuentes en los respectivos hospitales. En algunos hospitales se estudió más de una IIH.

Por tratarse de estudios pareados con muestras pequeñas, para calcular tanto el exceso de estadía como de utilización de antimicrobianos, se utilizó la prueba "Sign Rank test de Wilcoxon", con un $\mathrm{p}<0,05$.

\section{Resultados}

Se presentan los datos de 9 estudios de infección urinaria asociada a cateterismo urinario a permanencia (ITU), 4 de herida operatoria en distintas cirugías (IHO), 6 de endometritis (END), 4 de neumonía asociada a ventilación mecánica (NEU), 5 de infecciones del torrente sanguíneo asociadas a cateterismo venoso central (ITS) y 7 de infecciones de herida operatoria en cesárea (IHOC). El rango de pareamiento de los casos y controles fue sobre el $80 \%$ y la mayor dificultad para encontrar controles, estuvo en neumonía asociada a ventilación mecánica e infección del torrente sanguíneo. En END y NEU sólo hay estudios de hospitales dependientes del MINSAL. Todos los estudios mostraron sobre estadías promedio de casi el doble en los pacientes con IIH respecto a los controles sin IIH y lo mismo en relación a la utilización de antimicrobianos (Tabla 1). Hubo diferencias en los resultados para un mismo sitio de infección entre hospitales (Tablas 2a y 2 b). Las mayores sobre estadías se observaron en ITS e IHO (Tablas 3 y 4) y las menores en END e IHOC (Tablas 5 y 6). En términos de diferencias de estadía entre pacientes con IIH y 
sin IIH, la mayor se observó en neumonía que llegó hasta 6,6 veces (Tabla 7). La mayor heterogeneidad en sobre estadía y uso de antimicrobianos se observó en IHO (Tabla 8), probablemente relacionada con los distintos tipos de cirugías estudiadas (cirugías asociadas a trauma, colecistectomías laparoscópicas e implantes de cadera). No se observaron diferencias significativas en los resultados de estadía y uso de antimicrobianos según tipo de hospital. La menor utilización observada de antimicrobianos del hospital 2 (Tabla 9) y del hospital 1 (Tabla 10), se explica por tratarse de población pediátrica. La mayoría de los estudios mostró diferencias estadísticamente significativas en ambas variables estudiadas. Los resultados de los diferentes estudios no se consolidaron por su heterogeneidad y para destacar esta variabilidad.

Tabla 1. Efecto de la infección intrahospitalaria sobre la duración de la estadía y prescripción de antimicrobianos, según tipo de IIH

\begin{tabular}{lcccc}
\hline IIH & $\begin{array}{c}\mathbf{N}^{\mathbf{0}} \text { de } \\
\text { estudios }\end{array}$ & $\begin{array}{c}\mathbf{N}^{\mathbf{0}} \text { de casos } / \\
\text { controles }\end{array}$ & $\begin{array}{c}\text { Exceso de estadía } \\
\text { (rango en días) }\end{array}$ & $\begin{array}{c}\text { Exceso de uso de antimicrobianos } \\
\text { (rango en DDD) }\end{array}$ \\
\hline ITU* & 9 & $107 / 107$ & 7,5 a 31,2 & 3,9 a 61,2 \\
IHO & 4 & $44 / 44$ & 13 a 49,3 & 2,9 a 113,9 \\
END & 5 & $91 / 91$ & 2,6 a 5,6 & 7,1 a 18,7 \\
NEU & 4 & $47 / 47$ & 8,1 a 44,7 & 28 a 73,5 \\
ITS & 5 & $52 / 52$ & 6,6 a 64,0 & 0,5 a 58,4 \\
IHOC & 7 & $124 / 124$ & 1,0 a 7,9 & 6,8 a 47,9 \\
\hline
\end{tabular}

*Abreviaturas en el texto.

Tabla 2a. Diferencia en los días de estada en infección del tracto urinario

\begin{tabular}{cccccrc}
\hline Hospital & $\begin{array}{c}\text { Días } \\
\text { casos }\end{array}$ & $\begin{array}{c}\text { Promedio } \\
\text { casos }\end{array}$ & $\begin{array}{c}\text { Días } \\
\text { controles }\end{array}$ & $\begin{array}{c}\text { Promedio } \\
\text { controles }\end{array}$ & $\begin{array}{c}\text { Diferencia } \\
\text { Días }\end{array}$ & $\begin{array}{c}\text { Diferencia } \\
\text { promedio }\end{array}$ \\
\hline 1 & 253 & 25,3 & 118 & 11,8 & 135 & $13,5^{*}$ \\
2 & 378 & 37,8 & 220 & 22 & 158 & $15,8^{*}$ \\
3 & 224 & 22,4 & 138 & 13,8 & 86 & $8,6^{*}$ \\
4 & 545 & 54,5 & 233 & 23,3 & 312 & $31,2^{*}$ \\
5 & 260 & 26 & 163 & 16,3 & 97 & $9,7^{*}$ \\
6 & 838 & 41,9 & 566 & 28,3 & 272 & $13,6^{*}$ \\
7 & 571 & 33,6 & 444 & 26,1 & 127 & $7,5^{*}$ \\
8 & 245 & 24,5 & 98 & 9,8 & 147 & $14,7^{*}$ \\
9 & 257 & 25,7 & 158 & 15,8 & 99 & $9,9^{*}$ \\
\hline
\end{tabular}

$* \mathrm{p}<0,05$

Tabla 2b. Diferencia en la prescripción de antimicrobianos (promedio DDD) en infección del tracto urinario

\begin{tabular}{cccc}
\hline Hospital & Antimicrobianos casos & Antimicrobianos controles & Diferencia \\
\hline 1 & 18,3 & 14,4 & 3,9 \\
2 & 33,3 & 17,8 & $15,5^{*}$ \\
3 & 20,7 & 5,5 & $15,2^{*}$ \\
4 & 70,7 & 10,6 & $60,1^{*}$ \\
5 & 53,3 & 0 & $53,3^{*}$ \\
6 & 25,0 & 16,2 & 8,8 \\
7 & 23,6 & 8,8 & $14,8^{*}$ \\
8 & 78,5 & 17,3 & $61,2^{*}$ \\
9 & 18,5 & 3,1 & $15,4^{*}$ \\
\hline
\end{tabular}

$* \mathrm{p}<0,05$ 
Tabla 3. Diferencia en los días de estada en infección del torrente sanguíneo

\begin{tabular}{ccccccc}
\hline Hospital & $\begin{array}{c}\text { Días } \\
\text { casos }\end{array}$ & $\begin{array}{c}\text { Promedio } \\
\text { casos }\end{array}$ & $\begin{array}{c}\text { Días } \\
\text { controles }\end{array}$ & $\begin{array}{c}\text { Promedio } \\
\text { controles }\end{array}$ & $\begin{array}{c}\text { Diferencia } \\
\text { Días }\end{array}$ & $\begin{array}{c}\text { Diferencia } \\
\text { promedio }\end{array}$ \\
\hline 1 & 407 & 40,7 & 258 & 25,8 & 149 & $14,9^{*}$ \\
2 & 92 & 9,2 & 26 & 2,6 & 66 & 6,6 \\
3 & 666 & 60,5 & 342 & 31 & 324 & $29,5^{*}$ \\
4 & 1.699 & 154,4 & 998 & 90,4 & 701 & $64,0^{*}$ \\
5 & 408 & 40,8 & 209 & 20,9 & 208 & $20,8^{*}$ \\
\hline
\end{tabular}

Tabla 4. Diferencia en los días de estada en infección de herida operatoria

\begin{tabular}{clcccccc}
\hline Hospital & Tipo cirugías & $\begin{array}{c}\text { Días } \\
\text { casos }\end{array}$ & $\begin{array}{c}\text { Promedio } \\
\text { casos }\end{array}$ & $\begin{array}{c}\text { Días } \\
\text { controles }\end{array}$ & $\begin{array}{c}\text { Promedio } \\
\text { controles }\end{array}$ & $\begin{array}{c}\text { Diferencia } \\
\text { Días }\end{array}$ & $\begin{array}{c}\text { Diferencia } \\
\text { promedio }\end{array}$ \\
\hline 1 & Trauma & 364 & 26 & 97 & 6,9 & 267 & $19,1^{*}$ \\
2 & Trauma & 515 & 51,5 & 240 & 24 & 275 & $27,5^{*}$ \\
3 & Implante cadera & 659 & 65,9 & 166 & 16,6 & 493 & $49,3^{*}$ \\
4 & Colecistectomía & 200 & 20 & 70 & 7 & 130 & $13^{*}$ \\
\hline
\end{tabular}

$* \mathrm{p}<0,05$

Tabla 5. Diferencia en los días de estada en endometritis

\begin{tabular}{ccccccc}
\hline Hospital & $\begin{array}{c}\text { Días } \\
\text { casos }\end{array}$ & $\begin{array}{c}\text { Promedio } \\
\text { casos }\end{array}$ & $\begin{array}{c}\text { Días } \\
\text { controles }\end{array}$ & $\begin{array}{c}\text { Promedio } \\
\text { controles }\end{array}$ & $\begin{array}{c}\text { Diferencia } \\
\text { Días }\end{array}$ & $\begin{array}{c}\text { Diferencia } \\
\text { promedio }\end{array}$ \\
\hline 1 & 86 & 5 & 44 & 2,4 & 42 & 2,6 \\
2 & 29 & 9,6 & 12 & 4 & 17 & 5,6 \\
3 & 138 & 6,9 & 49 & 2,4 & 89 & $4,5^{*}$ \\
4 & 45 & 4,5 & 17 & 1,7 & 28 & 2,8 \\
5 & 204 & 6,8 & 111 & 3,7 & 93 & $3,1^{*}$ \\
6 & 99 & 9 & 39 & 3,6 & 60 & 5,5 \\
\hline
\end{tabular}

${ }^{*} \mathrm{p}<0,05$

Tabla 6. Diferencia en los días de estada en infección de herida operatoria cesárea

\begin{tabular}{ccccccc}
\hline Hospital & $\begin{array}{c}\text { Días } \\
\text { casos }\end{array}$ & $\begin{array}{c}\text { Promedio } \\
\text { casos }\end{array}$ & $\begin{array}{c}\text { Días } \\
\text { controles }\end{array}$ & $\begin{array}{c}\text { Promedio } \\
\text { controles }\end{array}$ & $\begin{array}{c}\text { Diferencia } \\
\text { Días }\end{array}$ & $\begin{array}{c}\text { Diferencia } \\
\text { promedio }\end{array}$ \\
\hline 1 & 97 & 9,7 & 47 & 4,7 & 50 & 5 \\
2 & 82 & 8,2 & 26 & 2,6 & 56 & 5,6 \\
3 & 138 & 6 & 83 & 3,6 & 55 & $2,4^{*}$ \\
4 & 81 & 8,1 & 53 & 5,3 & 28 & 2,8 \\
5 & 425 & 11,8 & 142 & 3,9 & 283 & $7,9^{*}$ \\
6 & 267 & 10,6 & 111 & 4,4 & 156 & $6,2^{*}$ \\
7 & 37 & 3,7 & 27 & 2,7 & 10 & 1,0 \\
\hline
\end{tabular}

*p $<0,05$

Tabla 7. Diferencia en los días de estada en neumonía

\begin{tabular}{ccccccc}
\hline Hospital & $\begin{array}{c}\text { Días } \\
\text { casos }\end{array}$ & $\begin{array}{c}\text { Promedio } \\
\text { casos }\end{array}$ & $\begin{array}{c}\text { Días } \\
\text { controles }\end{array}$ & $\begin{array}{c}\text { Promedio } \\
\text { controles }\end{array}$ & $\begin{array}{c}\text { Diferencia } \\
\text { Días }\end{array}$ & $\begin{array}{c}\text { Diferencia } \\
\text { promedio }\end{array}$ \\
\hline 1 & 458 & 45,8 & 69 & 6,9 & 389 & $38,9^{*}$ \\
2 & 301 & 30,1 & 106 & 10,6 & 195 & $19,5^{*}$ \\
3 & 583 & 34,2 & 261 & 26,1 & 322 & 8,1 \\
4 & 738 & 67,1 & 246 & 22,4 & 492 & $44,7^{*}$ \\
\hline
\end{tabular}

$* \mathrm{p}<0,05$ 
Tabla 8. Diferencia en la prescripción de antimicrobianos (promedio DDD) en infección de herida operatoria

\begin{tabular}{cccc}
\hline Hospital & Antimicrobianos casos & Antimicrobianos controles & Diferencia \\
\hline 1 & 125,2 & 11,3 & $113,9^{*}$ \\
2 & 91,2 & 2,9 & $88,3^{*}$ \\
3 & 39,6 & 6,1 & $33,5^{*}$ \\
4 & 3,3 & 0,4 & 2,9 \\
\hline
\end{tabular}

$* \mathrm{p}<0,05$

Tabla 9. Diferencia en la prescripción de antimicrobianos (promedio DDD) en neumonía

\begin{tabular}{cccc}
\hline Hospital & Antimicrobianos casos & Antimicrobianos controles & Diferencia \\
\hline 1 & 58,3 & 10,2 & $48,1^{*}$ \\
2 & 42 & 14 & 28 \\
3 & 81 & 7,5 & $73,5^{*}$ \\
4 & 87,4 & 21,7 & $65,7^{*}$ \\
\hline
\end{tabular}

Tabla 10. Diferencia en la prescripción de antimicrobianos (promedio DDD) en infección del torrente sanguíneo

\begin{tabular}{cccc}
\hline Hospital & Antimicrobianos casos & Antimicrobianos controles & Diferencia \\
\hline 1 & 10,3 & 9,8 & 0,5 \\
2 & 32,1 & 4,4 & $27,7^{*}$ \\
3 & 64,8 & 23,5 & $41,3 *$ \\
4 & 64,7 & 6,3 & $58,4^{*}$ \\
5 & 21,7 & 11,4 & 10,3 \\
\hline
\end{tabular}

$* \mathrm{p}<0,05$

Tabla 11. Diferencia antimicrobianos (promedio DDD) en infección de herida operatoria cesárea

\begin{tabular}{cccc}
\hline Hospital & Antimicrobianos casos & Antimicrobianos controles & Diferencia \\
\hline 1 & 54,3 & 6,4 & $47,9^{*}$ \\
2 & 35,2 & 0,1 & $35,1^{*}$ \\
3 & 47,9 & 0 & $47,9^{*}$ \\
4 & 14,2 & 1 & 13,2 \\
5 & 12,5 & 0,2 & 12,3 \\
6 & 37,9 & 4,3 & $33,6^{*}$ \\
7 & 7,6 & 0,8 & 6,8 \\
\hline
\end{tabular}

$* \mathrm{p}<0,05$

\section{Discusión}

Estimar los verdaderos costos en IIH es complejo y pueden están subestimados debido a que los estudios sólo consideran los costos directos de la atención. Sin embargo, con todas estas limitaciones, este tipo de investigación permite visualizar la enorme magnitud de este problema.

Los resultados de los diferentes estudios presentados corroboran los resultados presentados previamente, en el sentido que las IIH producen un aumento significativo de la estadía hospitalaria y de la utilización de antimicrobianos. En nuestro medio la sobre estadía observada es mayor que la publicada en E.U.A., probablemente relacionada con los sistemas de pago, ya que en general en Chile los financiadores (FONASA e ISAPRES) cancelan a los hospitales y clínicas por las prestaciones entregadas, en vez de un monto fijo por patologías específicas como es habitualmente en E.U.A. En este contexto en nuestro medio es el paciente o quien financia el que asume el mayor costo de las IIH.

Aunque no se han mostrado datos en indica- 
dores monetarios, en los estudios presentados el mayor peso corresponde a la estadía hospitalaria, que puede representar el $70 \%$ o más de los costos directos de las IIH. Tanto en NEU como en ITS una parte importante de la sobre estadía se produjo en unidades de pacientes críticos, lo que aumenta aún más el costo de este componente dado que en infecciones de estas localizaciones, la hospitalización en unidades críticas puede representar más de la mitad de la estadía ${ }^{11}$.

Aun cuando las menores estadías y utilización de antimicrobianos se observa en END e IHOC, estas infecciones son frecuentes y pueden presentarse tanto en hospitales grandes y complejos como en aquellos de menor complejidad.

Estos estudios demuestran el gran impacto económico que representan las IIH en el país y la importancia de mantener programas de prevención, que aun mínimamente desarrollados, son costo-efectivos ${ }^{1,12}$.

\section{Conclusiones}

Las IIH aumentan la estadía y uso de antimicrobianos entre 2 y 4 veces. La mayor sobre estadía se observó en las IHO e ITS y la mayor utilización de antimicrobianos fue en las IHO y NEU. No se observaron diferencias en los resultados entre hospitales a excepción de la infección de la herida operatoria debido probablemente a las distintas cirugías estudiadas.

\section{Resumen}

Este trabajo tiene como objetivo mostrar los resultados de 34 estudios de costos de infecciones intrahospitalarias (IIH) realizados en infección urinaria (ITU), herida operatoria (IHO), endometritis (END), neumonía (NEU), infecciones del torrente sanguíneo (ITS) y herida operatoria cesárea (IHOC), realizados en 24 hospitales de alta y mediana complejidad, tanto públicos como privados del país, utilizando la metodología propuesta por la OPS, en los que se compararon pacientes con IIH con otros pacientes sin IIH de igual sexo, grupo etario, patología y servicio clínico, en hospitales. Se calculó la estadía hospitalaria y la utilización de antimicrobianos en ambos grupos. Conclusiones: todos los estudios mostraron aumento de la estadía y de la utilización de antimicrobianos en los pacientes con IIH en rangos de entre 2 y 4 veces. Las IHO e ITS aumentan más la estadía que otras IIH. Las IHO y NEU aumentan más el uso de antimicrobianos que otras IIH. No hay grandes diferencias en los resultados por tipo de hospital. La mayor diferencia se observó en IHO, probablemente por los distintos tipos de cirugías estudiadas.

\section{Agradecimientos}

Agradecemos a los siguientes alumnos del Magíster en Ciencias Médicas, mención en Infecciones Intrahospitalarias y Epidemiología Hospitalaria de la Universidad de Valparaíso, por facilitar la información generada en los estudios realizados en los distintos hospitales y clínicas.

Mario Aguilera, Hugo Aranda, M. Angélica Apablaza, Inés Arribas, Jacqueline Astudillo, M. Teresa Caballero, Eliana Chacón, Mayling Chang, M. Isabel Collarte, Mónica Ferrada, Cecilia Gajardo, M. Eliana Gómez, Nerea González, Patricia González, Wilma Guzmán, Julia Hernández, Rosa Herrera, Carmen Laytte, Patricia Leiva, Patricia López, Wilma Medina, Juan Mendoza, Rodrigo Monsalve, Pilar Muñoz, Yolanda Parada, Mónica Pohlenz, Andrés Scherson, Marcos Sepúlveda, José Sovier, Marianella Teke, Vjera Triantafilo, Jenny Ulloa, Belia Vidal, Eduardo Wulff y Pilar Zárraga.

\section{Bibliografía}

1.- Jarvis W R. Selected aspect of the socioeconomic impact of nosocomial infections: morbidity, mortality, cost and prevention. Infect Control Hosp Epidemiol 1996; 17: 552-7.

2.- Haley R W. Cost-benefit analysis of infection control programs. Bennett J \& Brachman P. Hospital Infection $4^{a}$ ed. Philadelphia Lippincott-Raven 1998, pag 249-67.

3.- Mundy L, Fraser V. Determining the cost-effectiveness of hospital epidemiology and infection control programs. Glen Mayhall, Hospital Epidemiology and Infection Control $2^{\mathrm{a}}$ ed Philadelphia Lippincott Williams \& Wilkins 1999. pag. 1437-43.

4.- Otaíza F, Brenner P. Informe de la Vigilancia de Infecciones Intrahospitalarias Ministerio de Salud, Chile 1993.

5.- Nercelles P, Guerra S, Herrera R et al. Repercusiones económicas de las IIH. Libro de resúmenes del I Congreso Panamericano de IIH, Viña del Mar 1995.

6.- Herrera R, Valenzuela M, Carvajal L et al. Costos de la infección de herida operatoria cesárea. Libro de resúmenes del VI Congreso Chileno de IIH. Pucón 1997.

7.- Nercelles P, Villarroel M L, Herrera R et al. Costos de la bacteriemia primaria en un hospital terciario. Libro de resúmenes del VII Congreso Chileno de IIH. La Serena 1998.

8.- Nercelles P, Villarroel M L, Herrera R et al. Costos de neumonía no asociada a procedimientos invasivos en un hospital terciario. Libro de resúmenes del IX Congreso Chileno de IIH. Puerto Varas 2000.

9.- OPS. Protocolo para determinar el costo de la infección intrahospitalaria OPS/HCP/HCT/16/00.

10.- Otaíza F, Brenner P. Sistema de Vigilancia de las Infecciones Intrahospitalarias. Ministerio de Salud 1998.

11.- Pittet D, Tarara D, Wenzel R. Nosocomial bloodstream infection in critically ill patients. Excess length of stay, extra cost, and attributable mortality. JAMA 1984; 272 (23): 1819-20.

12.- Boyce J M, Potter-Bynoe G, Dziobek L. Nosocomial pneumonia in Medicare patients. Hospital cost and reimbursement patters under the prospective payment system. Arch Intern Med 1991; 151 (6): 1109-14.

Correspondencia a:

Pola Brenner Friedmann

E-mail: pbrenner@minsal.cl 